\title{
Smart Decision Support Systems for Volcanic Applications
}

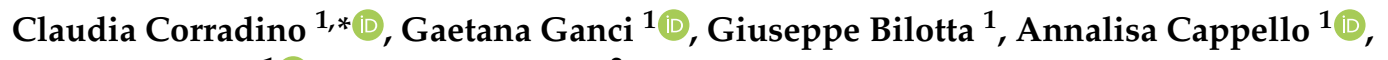 \\ Ciro Del Negro ${ }^{1}\left(\mathbb{D}\right.$ and Luigi Fortuna ${ }^{2}$ \\ 1 Istituto Nazionale di Geofisica e Vulcanologia, Sezione di Catania, 95125 Catania, Italy; \\ gaetana.ganci@ingv.it (G.G.); giuseppe.bilotta@ingv.it (G.B.); annalisa.cappello@ingv.it (A.C.); \\ ciro.delnegro@ingv.it (C.D.N.) \\ 2 Dipartimento di Ingegneria Elettrica Elettronica e Informatica, University of Catania, 95125 Catania, Italy; \\ luigi.fortuna@unict.it \\ * Correspondence: claudia.corradino@ingv.it
}

Received: 4 March 2019; Accepted: 25 March 2019; Published: 28 March 2019

\begin{abstract}
The huge amount of information coming from remote sensors on satellites has allowed monitoring changes in the planetary environment from about 50 years. These instruments are widely adopted to observe extreme thermal events such as eruptive phenomena in volcanic areas. Although the availability of so many different infrared sensors makes these instruments suitable to observe different kind of thermal phenomena, choosing the right infrared sensor to monitor each thermal event is not straightforward. In fact, the decision should take into account both the main features of the phenomena under investigation, e.g., its size and temperatures, that are often not known a priori, and the instruments specifications, e.g., spatial resolution. Here, a smart decision support system (SDSS) is proposed to address this task. In particular, we used a SDSS to simulate remote sensors responses, collect data coming from three different classes of remote sensors, retrieve information about the main features of the observed thermal event and, consequently, select the most suitable infrared remote sensor for the specific observed phenomena. Results obtained for a real case of study at Etna volcano is shown.
\end{abstract}

Keywords: remote sensing; smart system; volcanic applications

\section{Introduction}

In recent decades, spaceborne imaging spectroscopy has represented one of the most powerful investigation tools to monitor the Earth's surface. In particular, infrared remote sensing has been extensively adopted to observe high-temperature events such as volcanic eruptions across the world [1-4] and to retrieve the main features of such phenomena [5-11]. In fact, remote sensing devices measure the radiation emitted from the monitored hot surfaces, thus providing a measure of their temperature and a useful instrument to get their spatial distribution [12]. The possibility to quickly retrieve accurate information about hazardous volcanic events is of big interest for the scientific community cause more precise descriptions more precise predictions on the future volcanic scenario. However, instrumental limits do not allow receiving accurate information from the remote sensors and, depending on the kind of the event, some of them may either not be detected at all or lead the sensor to saturate. Furthermore, the existence of several remote sensors with different features, makes the choice of the sensor to be used neither easy nor fast.

The main technical features of these sensors are: spatial resolution, i.e., the area in the Earth's surface that a sensor can detect for each pixel; spectral resolution, i.e., the capability of a sensor to discriminate different bands in the electromagnetic spectrum; temporal resolution, i.e., the smallest 
time interval between two recordings covering exactly the same area; temperature limits, i.e., mainly determined by the limited dynamic range of a sensor, saturation temperature and the viewing pixel size; orbits, related to how the sensor satellite moves respect to the Earth, namely geostationary satellites and Sun-synchronous satellites. It is not possible to have an optimal device with all the best technical features at once [13]. Thus, the choice of the right sensors to use is not straightforward and depends on the specific case under investigation.

The effectiveness of multispectral observations of volcanic thermal features, e.g. lava lakes, active lava flows, magmatic intrusive bodies, has been shown in recent decades [14-20]. The emission of lava during volcanic eruptions is associated with temperatures between $800-1600 \mathrm{~K}$. In this wide thermal range, a great variability in size of the monitored volcanic features is observed, from small-scale active lava flows of the order of meters to large-scale active lava flows of the order of hundred meters. As soon as lava is ejected, it starts cooling turning into crust [21]. Thus, besides a very tiny area where temperature reaches nearly $1600 \mathrm{~K}$, the remaining cooling lava covers the greater proportion of the area with a temperature in the range of (350-1100K) [10]. When a volcanic zone is imaged by a remote sensor, the radiance emitted from the whole area covered by a pixel is converted to a unique integrated value resulting from the contributions of the sub-pixel components [12]. As a consequence, even the most accurate sensor, cannot give very precise information on the size and temperature of the thermal anomaly because of the limited spatial resolution. Generally speaking, thermal phenomena typically occupy a big sub-pixel portion in high spatial resolution payloads as VIIRS (Visible Infrared Imaging Radiometer Suite) I-bands, providing at-nadir pixel footprint of $375 \mathrm{~m}$; significant sub-pixel portion in moderate spatial resolution remote sensors, as SLSTR (Sea and Land Surface Temperature Radiometer) on board of SENTINEL-3, MODIS (Moderate Resolution Imaging Spectroradiometer) and AVHRR (Advanced Very High Resolution Radiometer); very small sub-pixel portion in low spatial resolution remote sensor as SEVIRI (Spinning Enhanced Visible and InfraRed Imager), onboard the geostationary Meteosat Second Generation MSG-1 to 4 [12]. From one hand, the higher the spatial resolution, the more details will be discernible, on the other hand using a device with an higher spatial resolution is not always the best choice. In fact, when thermal anomalies occur, an higher spatial resolution sensor has a greater proportion of sub-pixel components at higher temperature, thus increasing the pixel probability to saturate. Thus, besides the spatial resolution, the other sensor properties previously stated have to be taken into account to know the most suitable device to use.

As a consequence, thermal events observed by mean of different spaceborne infrared remote sensors, might or might not be detected either because of the monitored event features or because of the instrument technical limits.

Few studies have shown the thermal anomaly detection capability of remote sensors as a function of both their technical features and event features [22,23]. In [24], a fire detection algorithm is shown to be able to detect even quite small and cool fires when using MODIS imagery. Moreover, a comprehensive unique study comparing the performance of the main sensors, including the newest ones (SENTINEL-3 SLSTR), when observing thermal anomalies of different size and temperature has not been proposed yet. In case of volcanic events, these are useful to know more about the kind of volcanic activity and, in case of effusive eruptions, to find important lava flow parameters, e.g effusion rate. Thus, a tool able to extract more detailed information about the monitored thermal event from all the available raw remote sensors measurements would be very helpful for volcanic applications. This tool could drive the choice of the most appropriate imagery to use for the specific application. Usually, it is not a trivial task especially when dealing with volcanic phenomena requiring a fast response to rapidly face eruptive emergencies.

Here, we design a smart decision support system (SDSS) able to test the capability of different remote sensors from low to high spatial resolution, including the most recent ones, in detecting and characterizing different kinds of volcanic thermal anomalies taking into account sub-pixel scales. To do that, the main remote sensors, usually adopted for volcanic applications, are virtually implemented. 
The SDSS is a novel smart system able to automatically retrieve information on the size and temperatures of the investigated thermal anomaly at sub-pixel level by looking just at the mid-infrared band. It is a very powerful tool allowing to make the most from the raw measurements acquired by the remote sensors, providing help to decide the most appropriate sensor for the specific application.

SDSS is proposed to discover more information, in terms of size and temperature, about the thermal event under investigation and consequently choose the remote sensor that is more suitable for the specific case.

The rest of the paper is organized as follow: in Section 2, the main concepts behind infrared remote sensing and spaceborne remote sensors are presented focusing on the features relevant in volcanic applications; in Section 3, the SDSS is described, in Section 4, a real case study is investigated, in Section 5 , a discussion on the main results is presented.

\section{Infrared Remote Sensing}

Everything above absolute zero emits infrared radiation and the amount of emitted energy depends on the emissivity of the surface and on its kinetic temperature. The Planck's law, defined in Equation (1), describes the spectral radiance of the electromagnetic radiation emitted by a blackbody as a function of its wavelength and temperature.

$$
L(\lambda, T)=\frac{2 h c^{2}}{\lambda^{5}} \frac{1}{e^{\frac{h c}{\lambda k T}}-1}
$$

where $L(\lambda, T)$ is the radiance in $\left(\mathrm{Wsr}^{-1} \mathrm{~m}^{-2} \mu \mathrm{m}^{-1}\right)$ emitted at the wavelength $\lambda$ and temperature $T$ in $(K), \mathrm{h}$ is the Planck constant $\left(6.63 \times 10^{-34} \mathrm{Js}\right), c$ is the speed of light $\left(3 \times 10^{8} \mathrm{~ms}^{-1}\right), k$ the Boltzmann constant $\left(1.38 \times 10^{-23} J K^{-1}\right)$. Infrared remote sensors rely on this principle, thus, by measuring the part of the spectral radiance, $L(\lambda, T)$ thermally radiated from the surface under investigation, its kinetic temperature $T$ can be derived by reversing Equation (1) obtaining Equation (2).

$$
T=\frac{h c}{k \lambda} \frac{1}{\ln \left(\frac{2 h c^{2} \lambda^{-5}}{L(\lambda, T)}+1\right)}
$$

The wavelength $\lambda$ has a crucial role in the computation of the emitted radiance; in fact, each black body at a certain temperature will have a dominant wavelength $\left(\lambda_{\max }\right)$ at which it will emit the maximum radiance. Moreover, the Wien's displacement law states that the temperature of a black body and the wavelength at which it reaches its maximum emission are inversely related. Thus, the hotter the surface, the shorter wavelength will dominate the emitted radiance, meaning that the bands in the short wavelength infrared (SWIR) are particularly suitable for monitoring the thermal anomalies associated with high temperature events (i.e., the volcanic activity). The waveband centered at $4 \mu \mathrm{m}$ comprises the wavelengths of peak emission for high-temperature volcanic heat sources, being a very useful channel for hot spot detection. The hot radiant source imaged by satellite remote sensors may occupy just a portion of the whole pixel, meaning that other thermal components co-exist in the same pixel covered area. Thus, starting from Equation (1), the spectral radiance from a single pixel covering an area containing multiple portions $i$ with different temperatures $T_{i}$ may be expressed as

$$
L_{\text {pixel }}\left(\lambda, T_{\text {int }}\right)=\sum_{i=1}^{n} p_{i} L\left(\lambda, T_{i}\right)
$$

where $T_{\text {int }}$ is the integrated temperature, $n$ is the total number of sub-components with different temperatures $T_{i}, p_{i}$ is the fractional area of each $i_{t h}$ component and $L\left(\lambda, T_{i}\right)$ the corresponding spectral radiance [25].

For instance, an area characterized by three sub-pixel isothermal components, $p_{h}, p_{c}, p_{b}$ with temperatures, $T_{h}, T_{c}, T_{b}$ would result in 


$$
L_{\text {pixel }}\left(\lambda, T_{\text {int }}\right)=p_{h} L\left(\lambda, T_{h}\right)+p_{c} L\left(\lambda, T_{c}\right)+p_{b} L\left(\lambda, T_{b}\right)
$$

Equation (4) is named the "three-components equation" [12] and it has been widely adopted to characterize the main features of the sub-pixel areas by exploiting different wavelengths [12]. Thus, the pixel-integrated thermal value is less than the actual temperature of the hottest detected area.

On the other hand, each sensor has its own saturation temperature thus limiting its dynamical capability and sensors with higher spatial resolution may have higher probabilities to saturate than the ones with a lower spatial resolution. Moreover, it was demonstrated that the presence of a sub-pixel-sized hot spot causes emission around $4 \mu \mathrm{m}$ to largely increase making infrared remote sensors suitable to be used to identify thermal anomalies. The capability of each remote sensor to identify thermal anomalies at smaller sub-pixel scale at different temperatures depends on their technical characteristics, thus a brief description of the main satellite sensors is provided.

\section{Satellite Sensors}

The field of remote sensing started developing when the first sensor instrument platform was carried on board of the first satellites in 1960s-1970s. Since then, infrared remote sensors onboard of multispectral payloads have been used to identify and quantitatively characterize thermally 'anomalous' areas. Thus, a brief description of the three main remote sensors classes is provided.

The first class is the AVHRR-class, whose name comes from the first polar payload platform, containing moderate spatial resolution sensors. AVHRR is a radiation-detection imager with four channels carried for the first time on TIROS-N satellite (launched October 1978) with a simple design with a single detector for each channel. AVHRR has a big drawback in having a limited mid-infrared (MIR) dynamics to detect thermal anomalies in $1 \mathrm{~km}^{2}$ pixels since it saturates at only 320-330 K. AVHRR was in fact slowly replaced by MODIS when polar satellites TERRA and AQUA were launched respectively in 1999 and 2002. In fact, MODIS is a key-instrument for moderate-resolution applications monitoring high-temperature targets having a better MIR and thermal infrared (TIR) dynamics. In fact, it has a higher saturation temperature with respect to AVHRR, especially for the channel 21 (bandwidth 3.929-3.989 $\mu \mathrm{m}$ ) whose saturation temperature is $500 \mathrm{~K}$. Upon the success of MODIS, a novel remote sensing instrument was designed with similar features. The SLSTR on-board SENTINEL-3 mission has been launched on-board of Sentinel 3A satellite into a Sun-synchronous orbit on 16 February 2016. SLSTR has nine spectral bands (550-12000 nm) and a dual-view scan with swath widths of $1420 \mathrm{~km}$ and $750 \mathrm{~km}$, in nadir and backwards directions respectively. Better performance with respect to its predecessors are new channels (including two channels dedicated to fire detection), and higher resolution in some channels. In particular, it has two dedicated channels to monitor high thermal events at $1 \mathrm{~km}^{2}$ resolution, i.e., F1 channel at $3.7 \mu \mathrm{m}$ is capable of detecting very high temperatures anomalies without saturation ( $650 \mathrm{~K})$.

The second class is the VIIRS class, which concerns the Visible Infrared Imaging Radiometer Suite (VIIRS). It is a high spatial resolution sensor on board the Suomi National Polar-orbiting Partnership (Suomi NPP) and NOAA-20 weather satellites launched on the 28 October 2011. The VIIRS instrument is a whiskbroom scanning radiometer providing a full global coverage every day. VIIRS has twenty-two spectral bands $(0.412 \mu \mathrm{m}-12.01 \mu \mathrm{m})$ and the spatial resolution depends on the band of the electromagnetic spectrum; the panchromatic DNB and other sixteen bands have a spatial resolution of $750 \mathrm{~m}$ at nadir, the remaining five bands have a spatial resolution of $375 \mathrm{~m}$ at nadir (I-bands, imaging resolution bands). Among the overall improvements, VIIRS has better spatial resolution and it provides twice the coverage of its predecessors with providing a global coverage twice a day with either $750 \mathrm{~m}$ resolution across its entire scan or $375 \mathrm{~m}$ resolution. Furthermore, it has a panchromatic channel (sensitive to visible and near-infrared wavelengths) Day-Night-Band (DNB) providing nighttime imagery.

The third class is the GOES-class made by geostationary multispectral payloads with low spatial resolution. Although devices belonging to this class have very low spatial resolution, they have 
very high temporal resolution, e.g., up to 96 images daily, and long term observation due to their meteorological use. One of the most innovative sensors belonging to this class is SEVIRI, an high temporal and spectral resolution sensor that was launched in 2004 onboard of MSG1. SEVIRI has twelve spectral bands, four visible and near infraRed and eight infraRed. Among the visible ones, an High-Resolution visible (HRV) channel has an higher spatial resolution at $1 \mathrm{~km}$. The remaining channels have low spatial resolution at $3 \mathrm{~km}$. A full image can be completed in about $12.5 \mathrm{~min}$.

The infrared remote sensors that are considered are the best sensors for each class: SEVIRI (GOES-class), VIIRS-375 (VIIRS class) and SENTINEL-3-SLSTR (AVHRR-class). The Table 1 shows the main sensor features, namely the spatial resolution, the saturation temperature for each sensor and the noise equivalent temperature difference (NetD). The NetD is defined as the remote sensor thermal sensitivity, i.e., the sensor capability to distinguish two similar thermal radiation values in the image. It is a measure for how well a thermal imaging detector is able to distinguish between very small differences in thermal radiation in the image.

Table 1. Main satellite sensors features.

\begin{tabular}{cccc}
\hline Sensor & Spatial Resolution & NetD & Saturation Temperature \\
\hline SEVIRI & $9 \mathrm{~km}^{2}$ & $0.09 \mathrm{~K}$ & $335 \mathrm{~K}$ \\
SENTINEL-3 SLSTR & $1 \mathrm{~km}^{2}$ & $0.08 \mathrm{~K}$ & $500 \mathrm{~K}$ \\
VIIRS-375 & $0.1406 \mathrm{~km}^{2}$ & $0.486 \mathrm{~K}$ & $358 \mathrm{~K}$ \\
\hline
\end{tabular}

It is noteworthy that although VIIRS-375 has the highest spatial resolution, its saturation temperature is low, meaning that even a moderate thermal event whose dimension is of the same order of the pixel area may lead to saturation. The same event imaged by SENTINEL-3 SLSTR having a moderate spatial resolution and a higher saturation temperature would not saturate instead.

\section{Smart Decision Support System}

The SDSS contains a multi-remote sensors simulator that, given a thermal input, gives as output the pixel temperature resulting from the integration of the sub-pixels components as the real sensor would measure. Thus, it simulates how each investigated remote sensor responds to the radiance emitted by a surface considering a pixel characterized by different thermal components. To do that, the main features of each sensor are considered, namely the pixel spatial resolution, saturation temperature, NetD.

The main goal of the SDSS is to retrieve information on the observed thermal phenomena starting from remote sensors measurements, thus being able to select the ones that are better suited for the specific case of study. The fundamental task to perform is building a response map associating each sensor thermal response to a set of coupled values, i.e. the sizes and temperatures of the thermal phenomena.

To do that, different simulated data are created by considering different portions of sub-pixel components and different intensities. We decided to simulate three-components, as explained in Section 2, in the thermal pixel structure, namely the background temperature $\mathrm{Tb}$ occupying the portion $\mathrm{pb}$ of each pixel, the crust temperature Tc occupying the portion pc of each pixel, the melted lava temperature Th occupying the portion ph of each pixel.

As hypothesis, since usually the fractional area ph is very small of the order of $p h=10^{-5}$ (almost $100 \mathrm{~m}^{2}$ ) at $\mathrm{Th}=1373 \mathrm{~K}$, we vary $\mathrm{pb}$ and consequently $\mathrm{pc}=1$-pb-ph considering $\mathrm{Tb}=295 \mathrm{~K}$ and varying Tc in the interval $(350 \mathrm{~K}-1100 \mathrm{~K})$. The area covered by the simulated sensor has been set to $9 \times 10^{6} \mathrm{~m}^{2}$, the SEVIRI pixel area at Nadir.

During the calibration phase, SDSS receives the simulated data and as a first step, depending on the spatial resolution of each sensor (Table 1), provides an integrated value following Equation (4). Thus, a map for each sensor linking each sensor response with the sizes and temperatures of different phenomena can be built. 
The second step takes into account the limits of each sensor in terms of both NetD and saturation level (Table 1). Furthermore, as hypothesis based on previous studies [24,26], a thermal event is supposed to be a thermal anomaly when the actual measured temperature is greater than $310 \mathrm{~K}$ (channel noise) [24]. As a consequence, a working region for each class sensor can be defined inside the previously obtained map. Figure 1 reports the outcomes obtained considering as an example the case in which all the sensors classes look at the same thermal anomalies. In particular, the temperatures for each sensor when varying the crust temperature and crust area in the intervals previously defined are shown together with the dashed lines delimiting each sensor working region.

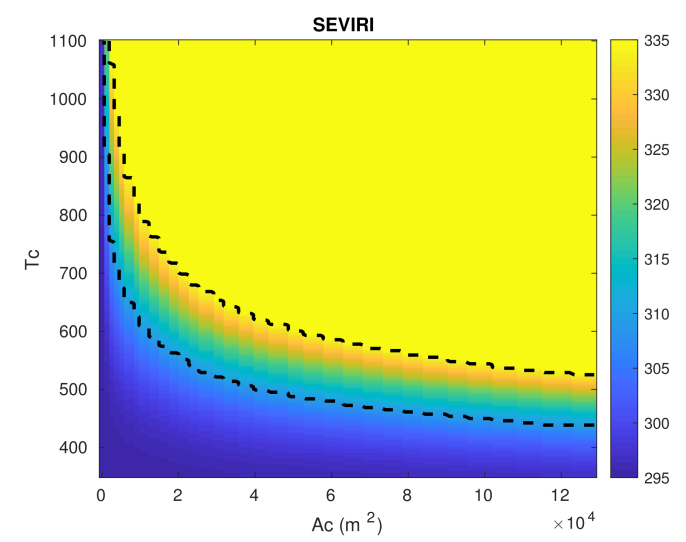

(a)

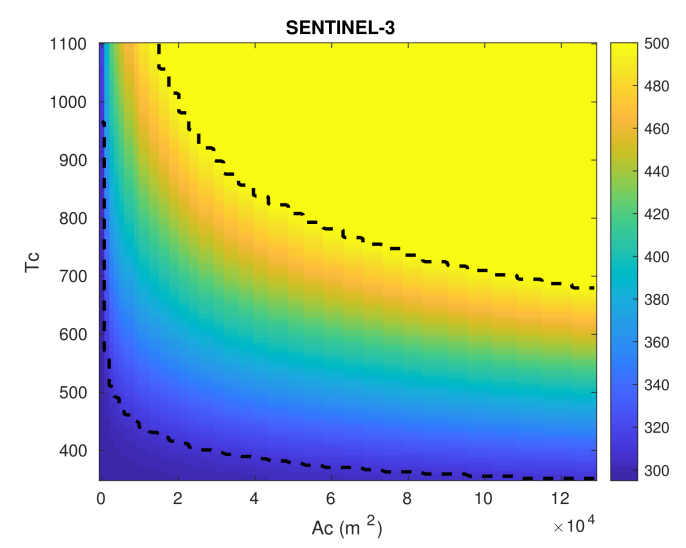

(b)

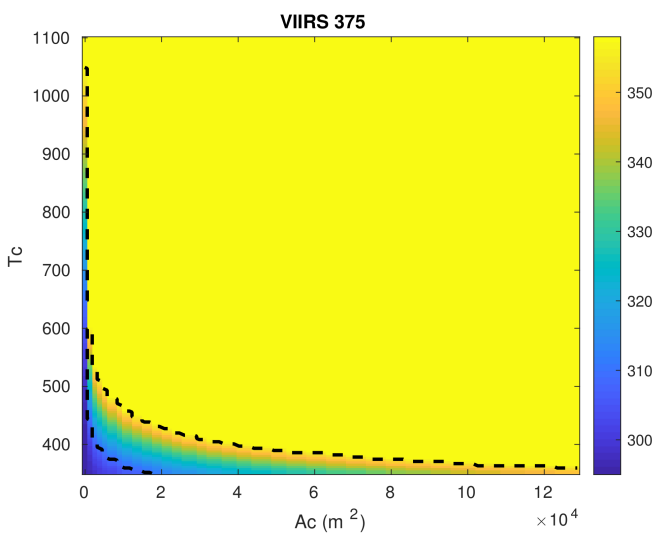

(c)

Figure 1. Simulated Response Map for each remote sensor: (a) SEVIRI; (b) SENTINEL-3 SLSTR; (c) VIIRS-375. The maximum area covered by the cooling lava $\left(A_{c}\right)$ corresponds to the VIIRS-375 pixel area $\left(14 \times 10^{4} \mathrm{~m}^{2}\right)$ less the hotter constant area set to $100 \mathrm{~m}^{2}$. 
Some considerations can be drawn from the obtained maps. It is noteworthy that although the GOESS-class (SEVIRI) has high temporal resolution thus being suitable for fast events monitoring, it has a small operative region where it performs well being not able to detect the smaller hot area. Viceversa, although AVHRR-class (SENTINEL-3 SLSTR and MODIS) has low temporal resolution preventing the detection of fast events, its operative region is wide with respect to the other sensors thus representing the best choice for slow thermal activities. In fact, it can detect small area even at higher temperature without saturating. On one hand, the performance of the moderate to high class comprising VIIRS-375 has a very good detection capability for small thermal areas despite the lower saturation temperature does not allow having a wide working area. On the other hand, they also have a good temporal resolution, thus being suitable for fast, small and thermal events in the low intensity window.

After the first calibration stage, the obtained maps are used by SDSS to retrieve information on the kind of event imaged by the remote sensors. Depending on the sensor thermal measurements, the system identifies a region in the parameters space $[A c, T c]$, i.e., the event typology, and consequently the most suitable sensors to use. In fact, the resulting parametric region, may or may not fall inside the sensors working regions previously defined (Figure 1) thus showing which sensor is more suitable to investigate each kind of event. In particular, the corresponding region in the parametric space $[A c, T c]$ is retrieved by the SDSS for each sensor estimating the size $(A c)$ and thermal range $(T c)$ of the event.

For instance, a large size low temperature event (e.g., $A c=127,300 \mathrm{~m}^{2}$ and $T c=365 \mathrm{~K}$ ) can be investigated with SENTINEL-3, while it is not seen by SEVIRI and VIIRS-375. In fact, in the first case, the pixel size is very big thus, although the thermal event size is quite big, being its temperature too low, the bigger proportion of the background sub-pixels makes the integrated pixel value too low to be detected; vice versa, in the latter case, since it is a smaller pixel, a greater part of that will be made by high thermal components, consequently it will saturate at a lower radiance. A small size low temperature event (e.g., $A c=27,210 \mathrm{~m}^{2}$ and $T c=365 \mathrm{~K}$ ) can be investigated only by using VIIRS-375 because only this very high resolution remote sensor is able to detect such a small and low thermal anomaly; a small size high temperature event (e.g., $A c=27,210 \mathrm{~m}^{2}$ and $T c=685 \mathrm{~K}$ ) can be investigated only by using SENTINEL-3 SLSTR since the others will saturate instead; a medium size average/high temperature event (e.g., $A c=67,510 \mathrm{~m}^{2}$ and $T c=534 \mathrm{~K}$ ) can be seen only by SENTINEL-3 SLSTR and SEVIRI since VIIRS-375 saturates.

The previous example gives an insight into the applicability of this system to the more general cases. Thus, SDSS helps understanding the kind of thermal event under investigation and, depending on this, helps choosing the most suitable sensor candidates to use.

\section{Results}

Now, we present a real case of study to test the SDSS. Firstly, the main sensors of the three classes are simulated with SDSS when monitoring a real volcanic event and the resulting integrated temperature for each sensor is computed. Secondly, the computed pixel integrated temperature is used to retrieve information on the size and real temperature of the volcanic phenomena by exploiting the simulated response maps obtained in the previous section. Finally, the best remote sensor for this specific application is identified.

The input data presented to the SDSS is taken from real measurements of a lava fountain and concurrent lava flow emplacement at Mount Etna, Sicily, recorded by a permanent thermal camera on the 12 August 2011 [27]. Data coming from this thermal camera is re-projected into a digital elevation model (DEM) and then given as an SDSS input (it is a simplification because we are supposing that the angle view is at nadir). The size of the considered area is $9 \times 10^{6} \mathrm{~m}^{2}$ and different time instants have been considered, namely 8:00, 10:30 and 23:00. SDSS will simulate the remote sensor response for each class-sensor as it is shown in Figure 2. 


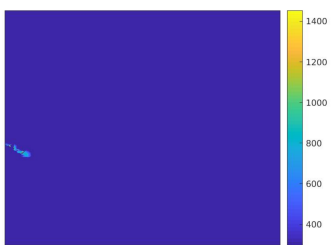

(a)

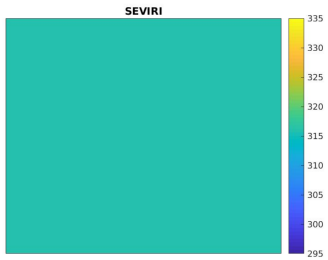

(d)

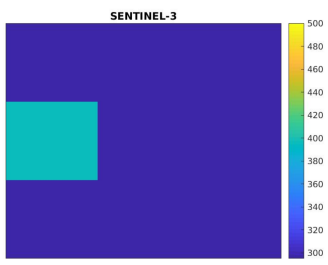

(g)

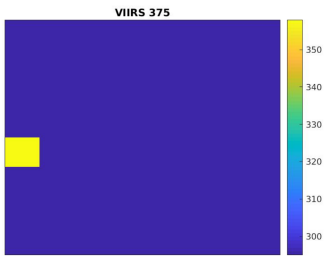

(j)

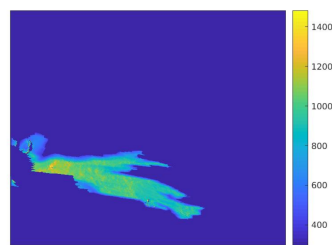

(b)

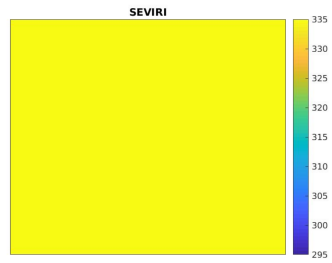

(e)

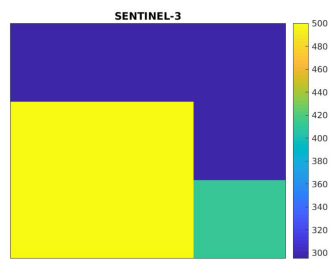

(h)

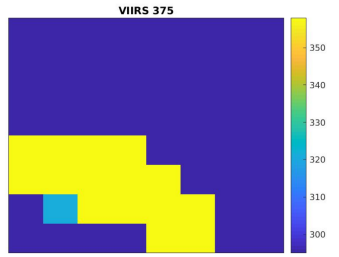

(k)

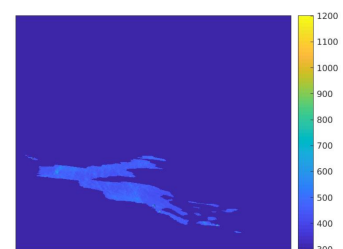

(c)

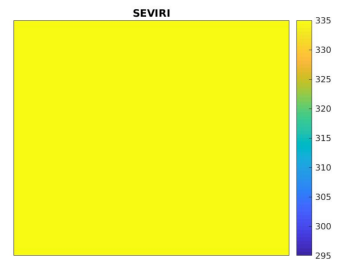

(f)

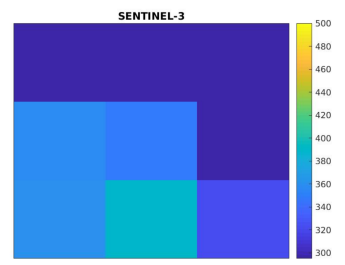

(i)

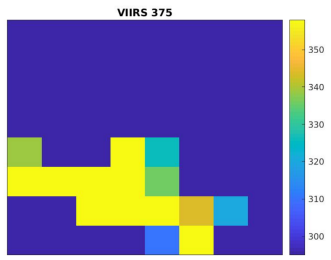

(l)

Figure 2. Remote sensor response for each class-sensor for the lava flow emplacement at Mount Etna, Sicily on the 12 August 2011 for each remote sensor : (d-f), SEVIRI; (g-i) SENTINEL-3 SLSTR; (j-1) VIIRS-375.

By only looking at the simulated sensors responses, a global consideration on the remote sensors performance can be drawn. For instance, it is noticeable that during the lava cooling phase shown in Figure 2c, SENTINEL-3 SLSTR is the best candidate cause it is the only one whose pixels do not saturate in the whole area covered by the volcanic deposits (Figure 2f,i,l).

Small sizes thermal anomalies are often difficult to be detected and at the same time are fundamental in terms of early warning. Thus, we focus on this case to test SDSS.

At 8:00, a very small thermal anomaly appears as the camera shows (Figure 2a). The three sensors simulated responses produced with SDSS are shown in Figure $2 \mathrm{~d}, \mathrm{~g}, \mathrm{j}$. It is noteworthy that the VIIRS-class is not well suited for this thermal event since VIIRS-375 saturates. In fact, this case has been listed in the previous section, when only SEVIRI and SENTINEL-3 SLSTR could be used.

At this point, we expect that knowing the integrated pixel value for each sensor, the simulated response map as the one shown in Figure 1 can be used to determine the area and the temperature of the monitored thermal event. Thus, considering the outcome of each SDSS simulated sensor, the parametric region $[A c, T c]$ of the lava flow emplacement at 8:00 is retrieved for each sensor as it is shown in Figure 3. The working region defined in the previous section for each sensor is graphed as the blue-white area, while the identified parametric region $[A c, T c]$ is shown as the yellow area. 


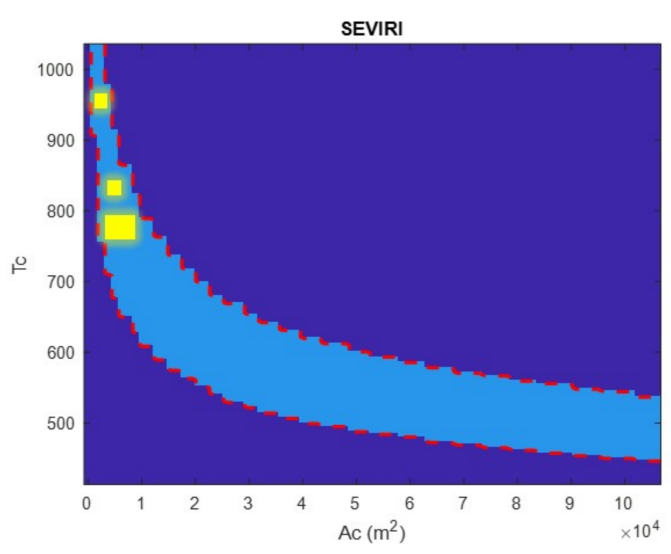

(a)

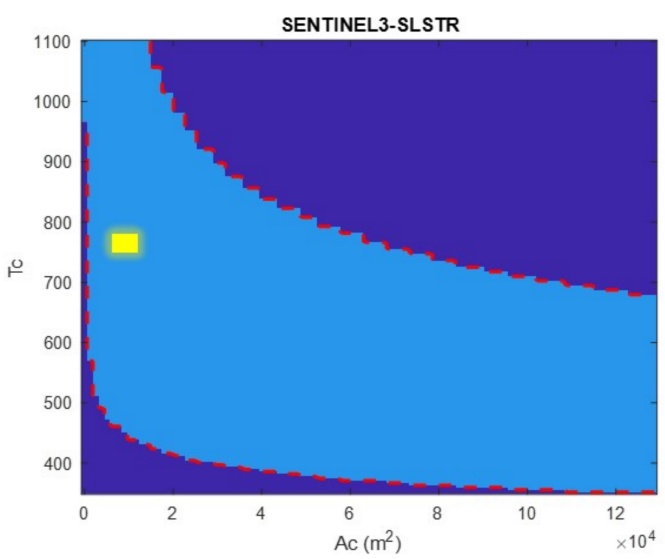

(b)

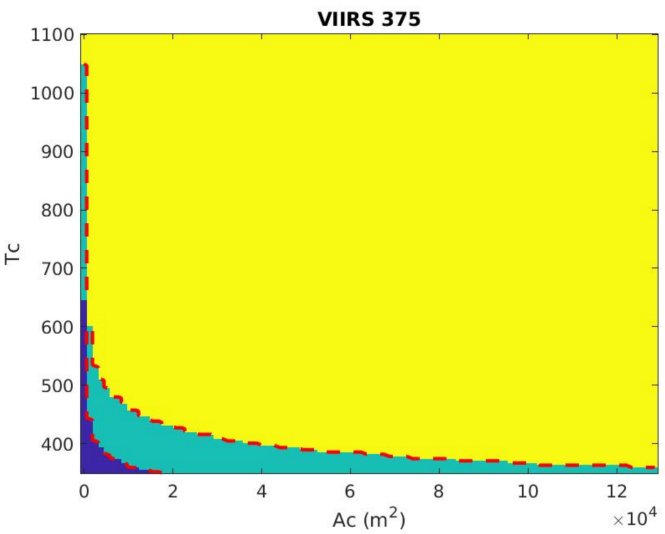

(c)

Figure 3. Retrieved regions $[A c, T c]$ for each remote sensor : (a) SEVIRI; (b) SENTINEL-3 SLSTR; (c) VIIRS-375.

As expected, VIIRS-375 saturates so the detected parametric region falls outside the sensor working region. In other words, this sensor cannot be used for this specific case because it does not give any relevant information about the event under investigation. As it is noticeable from Figure 3c, it could be any kind of thermal anomaly, e.g., a small and hot thermal event but also a very big and cold thermal event. On the other hand, SEVIRI will identify three possible parametric regions associated with the known integrated temperature. SENTINEL-3 SLSTR identifies a unique retrieved region $[A c, T c]$ that coincides with one of the regions previously found by SEVIRI simulator. Thus, thanks to the synergistic use of SEVIRI and SENTINEL-3 SLSTR, SDSS is able to provide a good 
description of the thermal event providing an estimation of the size and temperature of the observed thermal phenomena.

It is important to notice that SDSS has been calibrated by using the three-components equation (Equation (4)). Although this approximation has been widely adopted in the past, in real cases more than three thermal sub-pixels components are present as in this case. Thus, this simplification may lead to an estimation error. Despite this, SDSS is able to estimate the range of the possible sizes and temperatures giving an insight into the kind of possible observed thermal event. Then, in this case it is possible to say that the main thermal component of the event has an average hot temperature in the range of $[700 \mathrm{~K}, 760 \mathrm{~K}]$ and a size in the range of $\left[8900 \mathrm{~m}^{2}, 11,000 \mathrm{~m}^{2}\right]$. As it can be noticed from Figure $2 \mathrm{a}$, the average hot temperature and the estimated area of the lava flow emplacement seem to fall in this range.

To estimate the error coming from the simplification of the three-components equation, we compare the radiance evaluated for each thermal component of the real case of study and the radiance computed by mean of the simplification performed by the SDSS. In particular, for the latter case, we set $p h$ and $T h$ to the default values, while we use a value inside the ranges found for $A c$ and $T c$, namely $A c=9010 \mathrm{~m}^{2}$ and $T c=757 \mathrm{~K}$. Thus, considering SENTINEL3-SLSTR spatial resolution, $p c=0.00901$ and consequently $p b=1-p c-p h=0.99098$. The radiance estimated by mean of this simplification is $L=9.5$ [W sr$\left.{ }^{-1} \mathrm{~m}^{-2} \mu \mathrm{m}^{-1}\right]$, while the radiance measured with the $\mathrm{n}$-components Equation (3) is $L=10.4\left[\mathrm{~W} \mathrm{sr}^{-1} \mathrm{~m}^{-2} \mu \mathrm{m}^{-1}\right]$. Thus, the error $\mathrm{e}=0.9\left[\mathrm{~W} \mathrm{sr}^{-1} \mathrm{~m}^{-2} \mu \mathrm{m}^{-1}\right]$ corresponding to a thermal error of about $3.7 \mathrm{~K}$, is due to the simplification of using three rather than $\mathrm{n}$ components.

\section{Discussion}

This new smart device support system fully exploits remote sensors capabilities and investigates even further the raw measurements provided by remote sensors.

SDSS simulates the responses of the three main remote multispectral sensors classes for thermal monitoring based on the real known spatial features. In particular, SDSS builds a simulated response map for each class showing the relationship between the area and the temperature of the monitored thermal event and the corresponding sensor integrated temperature. Thus, it compares the performance of different remote sensors being able to know which sensor is more suitable for the specific case. In particular, from the achieved results, it is worth noticing that SENTINEL-3 SLSTR is the satellite sensor offering the best performance with respect to the other class sensors being characterized by a wider working region. Even though SENTINEL3-SLSTR temporal resolution is low, the progress running in the last few years paves the way to having a higher and higher temporal resolution for the new upcoming moderate spatial resolution class sensors.

The SDSS parametric maps allow retrieving information on the observed thermal event in terms of size and temperature starting from a known temperature sensor measurement; the real case previously analyzed, clearly shows the advantage of using this approach. In particular, the real small volcanic event in Figure 2 that is characterized by several thermal components (from $600 \mathrm{~K}$ upwards), is perceived in different ways by the three different remote sensor classes. They all underestimate the thermal event temperature providing low integrated value at the pixel level, namely nearly $400 \mathrm{~K}$ for SENTINEL-3 SLSTR, $315 \mathrm{~K}$ for SEVIRI and $358 \mathrm{~K}$ (saturation temperature) for VIIRS-375. VIIRS-375 response saturates cause the event area cover a big fraction of the pixel and the event temperature is quite high with respect to the sensor saturation temperature thus leading to the pixel saturation. Then, apart from this case that cannot be used for further analysis, the other sensors widely underestimate the real temperature cause a big fraction of the sub-pixel components is at low temperature, i.e., the background temperature. Despite this, as Figure 3c shows, the SDSS is able to rightly describe the monitored event as a small volcanic phenomena in the range of $\left[8900 \mathrm{~m}^{2}, 11000 \mathrm{~m}^{2}\right]$ with temperature in the range of $[700 \mathrm{~K}, 760 \mathrm{~K}]$. In other words, from the unique integrated pixel value provided by each remote sensor, the SDSS gives two output: a more accurate measurement of the monitored volcanic phenomena and an estimation of the size of such thermal event. 
To validate the SDSS, data coming from an installed thermal camera are used providing information on the real monitored thermal event in terms of size and temperature. Since this instrument is often not available during eruptive activity, adopting the SDSS allows making an estimation on the kind of the investigated phenomena. In fact, without knowing anything about the thermal event, the SDSS outcomes allow describing the monitored volcanic activity.

For instance, by only looking at the SDSS response provided in Figure 3b, it is possible to state that the event represents the very early onset of a volcanic eruption or, in general, a strombolian activity. Moreover, the SDSS response associated with the case in Figure $2 b$ would suggest that the monitored event represents the formation of an active lava flow; while the SDSS response associated with the case in Figure 2c would suggest that the the cooling phase of a lava flow is taking place. This can be used to know more about the kind of event, to monitor the volcanic eruption evolution and to stay updated on the current state of the monitored activity.

Furthermore, it would be possible to narrow even more the parametric region defining the kind of volcanic event by exploiting more bands. In fact, SDSS uses only the MIR band while providing very good results. The same approach could be applied to more bands thus adding even more precision to the final estimates.

\section{Conclusions}

When monitoring volcanic thermal phenomena, it is fundamental to be able to retrieve their most accurate description in terms of size and temperature. In fact, this information is needed both to derive other important volcanic parameters and to rapidly face eventual eruptive emergencies. However, instrumental limits lead to inaccurate remote sensor measurements and consequently, errors in thermal anomaly detection and characterization. Furthermore, the existence of several remote sensors with different features, makes the choice of the sensor to be used neither easy nor fast.

Starting from these considerations, we proposed a SDSS tool able not only to provide a comparison of the main sensors performance, including the recent ones (e.g., SENTINEL-3 SLSTR), when observing volcanic phenomena of different sizes and temperatures, but also to retrieve more accurate information on the observed phenomena at sub-pixel level and consequently, to identify the best sensor to use for the specific target. The investigation of the real case of study highlighted that the sensor that is more suited for volcanic application is SENTINEL-3 SLSTR, in fact it allows retrieving more accurate information on the thermal event size and temperature with respect to the other sensors with a wider working region.

A comprehensive comparison of the main satellite remote sensors (including the most recent ones) for investigations of high-temperature volcanic features has not been proposed yet. Furthermore, SDSS is the first smart system able to retrieve important information about the investigated thermal features at sub-pixel level and thus support is provided for deciding the most appropriate sensor for the specific application. SDSS is a very powerful tool allowing to make the most from the raw measurements provided by the remote sensors, extracting two accurate information on the volcanic phenomena (size and temperature) that are fundamental for volcanic applications.

It is important to notice that at this phase, the temporal resolution of the available sensors is not taken into account, thus the future work will be devoted to take into account the temporal resolution. The big limitation at the moment is that the SDSS does consider the approximation of the three-components equation thus making a big simplification, despite the provided results show that the error is not too big. Furthermore, the satellite sensors are not synchronized yet, meaning that the images that are compared by the SDSS are not recorded at exactly the same time ( they have a small delay). However, the future trend is going towards the synchronization of these sensors thus overcoming this limit. 
Author Contributions: Conceptualization, C.C. and G.G.; methodology, C.C. and G.G.; software, C.C.; validation, G.B.; formal analysis, A.C.; investigation, C.C. and G.G; resources, A.C.; data curation, G.B; writing-original draft preparation, C.C., G.G and C.D.N.; writing-review and editing, C.C., G.G, A.C., G.B., C.D.N. and L.F.; visualization, L.F. and C.D.N.; supervision, L.F. and C.D.N.; project administration, C.D.N.; funding acquisition, C.D.N.

Funding: This research was partially funded by ATHOS Research Programme.

Acknowledgments: This work was developed within the framework of TecnoLab, the Laboratory for Technological Advance in Volcano Geophysics of the INGV in Catania (Italy). We thank the Editor and two anonymous reviewers for the constructive and supportive comments that helped to improve the manuscript.

Conflicts of Interest: The authors declare no conflict of interest.

\section{References}

1. Ganci, G.; Bilotta, G.; Cappello, A.; Herault, A.; Del Negro, C. HOTSAT: A multiplatform system for the thermal monitoring of volcanic activity using satellite data. Geol. Soc. Lond. Spec. Publ. 2016, 426, $207-221$. [CrossRef]

2. Patrick, M.R.; Kauahikaua, J.; Orr, T.; Davies, A.; Ramsey, M. Operational thermal remote sensing and lava flow monitoring at the Hawaiian Volcano Observatory. Geol. Soc. Lond. Spec. Publ. 2016, 426, 489-503. [CrossRef]

3. Cappello, A.; Ganci, G.; Bilotta, G.; Herault, A.; Zago, V.; Del Negro, C. Satellite-driven modeling approach for monitoring lava flow hazards during the 2017 Etna eruption. Ann. Geophys. 2018, 61, 13. [CrossRef]

4. Pergola, N.; D’Angelo, G.; Lisi, M.; Marchese, F.; Mazzeo, G.; Tramutoli, V. Time domain analysis of robust satellite techniques (RST) for near real-time monitoring of active volcanoes and thermal precursor identification. Phys. Chem. Earth 2009, 34, 380-385. [CrossRef]

5. Ganci, G.; Cappello, A.; Bilotta, G.; Herault, A.; Zago, V.; Del Negro, C. Mapping volcanic deposits of the 2011-2015 Etna eruptive events using satellite remote sensing. Front. Earth Sci. 2018, 6, 83. [CrossRef]

6. Slatcher, N.; James, M.; Calvari, S.; Ganci, G.; Browning, J. Quantifying effusion rates at active volcanoes through integrated time-lapse laser scanning and photography. Remote Sens. 2015, 7, 14967-14987. [CrossRef]

7. Wooster, M.J.; Roberts, G.; Perry, G.L.W.; Kaufman, Y.J. Retrieval of biomass combustion rates and totals from fire radiative power observations: FRP derivation and calibration relationships between biomass consumption and fire radiative energy release. J. Geophys. Res. Atmos. 2005, 110, 311. [CrossRef]

8. Wooster, M.J.; Zhukov, B.; Oertel, D. Fire radiative energy for quantitative study of biomass burning: Derivation from the BIRD experimental satellite and comparison to MODIS fire products. Remote Sens. Environ. 2003, 86, 83-107. [CrossRef]

9. Zakšek, K.; Hort, M.; Lorenz, E. Satellite and ground based thermal observation of the 2014 effusive eruption at Stromboli volcano. Remote Sens. 2015, 7, 17190-17211. [CrossRef]

10. Vulpiani, G.; Ripepe, M.; Valade, S. Mass discharge rate retrieval combining weather radar and thermal camera observations. J. Geophys. Res. Solid Earth 2016, 121, 5679-5695. [CrossRef]

11. Bato, M.G.; Froger, J.L.; Harris, A.J.L.; Villeneuve, N. Monitoring an effusive eruption at Piton de la Fournaise using radar and thermal infrared remote sensing data: insights into the October 2010 eruption and its lava flows. Geol. Soc. Lond. Spec. Publ. 2016, 426, 533-552. [CrossRef]

12. Harris, A. Thermal Remote Sensing of Active Volcanoes: A User's Manual; Cambridge University Press: Cambridge, UK, 2013.

13. Blackett, M. An overview of infrared remote sensing of volcanic activity. J. Imaging 2017, 3, 13. [CrossRef]

14. Del Negro, C.; Cappello, A.; Ganci, G. Quantifying lava flow hazards in response to effusive eruption. GSA Bull. 2016, 128, 752-763. [CrossRef]

15. Harris, A.J.L.; Vaughan, R.A.; Rothery, D.A. Volcano detection and monitoring using AVHRR data: The Krafla eruption, 1984. Remote Sens. 1995, 16, 1001-1020. [CrossRef]

16. Ganci, G.; Cappello, A.; Zago, V.; Bilotta, G.; Herault, A.; Del Negro, C. 3D Lava flow mapping of the 17-25 May 2016 Etna eruption using tri-stereo optical satellite data. Ann. Geophys. 2018, 61, 15. [CrossRef]

17. Cappello, A.; Ganci, G.; Calvari, S.; Pérez, N.M.; Hernández, P.A.; Silva, S.V.; Cabral J.; Del Negro, C. Lava flow hazard modeling during the 2014-2015 Fogo eruption, Cape Verde. J. Geophys. Res. Solid Earth 2016, 121, 2290-2303. [CrossRef] 
18. Coppola, D.; Laiolo, M.; Cigolini, C.; Delle Donne, D.; Ripepe, M. Enhanced volcanic hot-spot detection using MODIS IR data: Results from the MIROVA system. Geolog. Soc. Lond. Spec. Publ. 2016, 426, 181-205. [CrossRef]

19. Ganci, G.; Vicari, A.; Cappello, A.; Del Negro, C. An emergent strategy for volcano hazard assessment: From thermal satellite monitoring to lava flow modeling. Remote Sens. Environ. 2012, 119, 197-207. [CrossRef]

20. Ferrucci, F.; Hirn, B. Automated monitoring of high-temperature volcanic features: From high-spatial to very-high-temporal resolution. Geol. Soc. Lond. Spec. Publ. 2016, 426, 159-179. [CrossRef]

21. Hirn, B.; Di Bartola, C.; Ferrucci, F. Synergetic exploitation of meteorological geostationary payloads «SEVIRI» and «JAMI» for quantitative, real-time, global volcano monitoring. In Proceedings of the IEEE International Geoscience and Remote Sensing Symposium, Honolulu, HI, USA, 25-30 July 2010; pp. 1549-1552.

22. Higgins, J.; Harris A. VAST: A program to locate and analyse volcanic thermal anomalies automatically from remotely sensed data. Comput. Geosci. 1997, 23, 627-645. [CrossRef]

23. Wooster, M.J.; Weidong X.; Nightingale T. Sentinel-3 SLSTR active fire detection and FRP product: Pre-launch algorithm development and performance evaluation using MODIS and ASTER datasets. Remote Sens. Environ. 2012, 120, 236-254. [CrossRef]

24. Giglio, L.; Schroeder, W.; Justice, C.O. The collection 6 MODIS active fire detection algorithm and fire products. Remote Sens. Environ. 2016, 178, 31-41. [CrossRef] [PubMed]

25. Murphy, S.W.; Oppenheimer C.; De Souza Filho, C.R. Calculating radiant flux from thermally mixed pixels using a spectral library. Remote Sens. Environ. 2014, 142, 83-94. [CrossRef]

26. Hua, L.; Shao, G. The progress of operational forest fire monitoring with infrared remote sensing. J. For. Res. 2017, 28, 215-229. [CrossRef]

27. Ganci, G.; James, M.R.; Calvari, S.; Del Negro, C. Separating the thermal fingerprints of lava flows and simultaneous lava fountaining using ground-based thermal camera and SEVIRI measurements. Geophys. Res. Lett. 2013, 40, 5058-5063. [CrossRef]

(C) 2019 by the authors. Licensee MDPI, Basel, Switzerland. This article is an open access article distributed under the terms and conditions of the Creative Commons Attribution (CC BY) license (http:// creativecommons.org/licenses/by/4.0/). 